\title{
Novel Correspondence-based Approach for Consistent Human Skeleton Extraction
}

\author{
Kang Wang ${ }^{1}$, Abdul Razzaq ${ }^{1}$, Zhongke Wu${ }^{* 1}$, Feng Tian ${ }^{2}$, Sajid Ali ${ }^{1}$, Taorui Jia ${ }^{1}$, \\ Mingquan Zhou' \\ ${ }^{1}$ Beijing Key Laboratory of Digital Preservation and Virtual Reality for Cultural Heritage, Beijing Normal University, P.R.C \\ ${ }^{2}$ School of Design, Engineering \& Computing, Bournemouth University, UK
}

\begin{abstract}
This paper presents a novel base-points-driven shape correspondence (BSC) approach to extract skeletons of articulated objects from 3D mesh shapes. The skeleton extraction based on BSC approach is more accurate than the traditional direct skeleton extraction methods. Since 3D shapes provide more geometric information, BSC offers the consistent information between the source shape and the target shapes. We first extract the skeleton from a template such as the source shape automatically. Then, the skeletons of the target shapes of different poses are generated based on the correspondence relationship with the source shape. The accuracy and effectiveness of the proposed method is demonstrated through a comprehensive performance evaluation on multiple benchmark datasets. The results of the proposed approach can be applied to various applications such as skeleton-driven animation, shape segmentation and human motion analysis.
\end{abstract}

Keywords Shape Correspondence $\cdot$ Heat Kernel Signature • Mesh Contraction • Skeleton Extraction

* Corresponding author
Beijing Normal University, China
e-mail: zwu@bnu.edu.cn(Z. Wu)

\section{Introduction}

The 3D shape correspondence is to find a meaningful relation between pair of shapes and it has a wide range of applications in a variety of domains, such as geometric modeling and processing [1], shape recognition [2, 3], shape registration and shape retrieval [4, 5]. The representation of variations of human shapes has been an important topic in computer vision and graphics. Establishing a significant relationship between shape correspondences of similar model with different poses is a challenging task for several reasons. Firstly, among the different types of poses from the same model such as a person, there are many non-rigid deformations [6]. Secondly, the motion capture system could capture 3D postures through multiple cameras environment [7], but we need the meaningful relationship of shape points among distinctive pose shapes.

The shape correspondence is also closely related to the extraction of the curve-skeleton of shape. An articulated 3D model skeleton provides an intuitive abstraction for both geometrical and topological shape of the objects. An extracted 1D curve-skeleton is an effective representation of the model, which facilitates the manipulation and understanding of the shape of model [8]. The skeletal representation of the model has become very popular and 
finds a wide range of applications in shape analysis [9], surface reconstruction [10], character skinning through skeletal rigging [11], skeleton-driven character animation [12], object matching and shape retrieval $[13,14]$ and shape deformation through skeleton [15]. With the capability of creating a three-dimensional representation of human models, the extraction of curve-skeletons for these models has become a fundamental problem in many applications.

A number of methods have been proposed to extract a skeleton from 3D shape [16,17]. Majority of these methods are geometry-based, which have a hybrid approach combining topology and geometry-based techniques [18]. An approach which has the combination of capabilities such as robust and high accuracy to extract a skeleton for different poses of same model is highly required. The extracted skeletons by using these methods are often not satisfactory due to undesirable redundant branches, complexity in joints hierarchy and manual approximations during extraction. Technically, in skeleton extraction of the shape, an extracted skeleton is of well-centered, well-define joint hierarchy joints. The accurate joint identification that matches the real position in every pose of the shape is a challenging task in computer graphics and interactive applications. A number of commercial 3D packages (such as Maya $^{\mathrm{TM}}$, Blender, etc.) have been developed for creating automatic skeleton for the object. However, the generation of skeleton by using these packages is time-consuming, laborintensive and the results to large extent depend on user's skills.

In this paper, we propose and develop an integrated framework of consistent human skeleton extraction based on the base-points-driven shape correspondence (BSC). The source and target shapes are topologically consistent, with different postures and semantically similar to each other. BSC establishes the corresponding relationship between two shapes using local maximum Scale-invariant Heat Kernel signatures as base points to embed the original shapes into a Euclidian space. In this space, we first compute the similarities between points on source and target shapes, and then build the correspondence, based on which the skeleton can be extracted.

To extract the skeleton of a source shape, at first, the source shape is contracted through Laplacian-based mesh contraction method. A 1D curve-skeleton of the contracted shape is then obtained through topological thinning. The final joint-based skeleton shape is produced by applying geometric refinements on extracted $1 \mathrm{D}$ curve-skeleton of the source shape. The consistent skeleton of the target shape which possesses different poses of the source shape is then generated based on correspondence relationship between these shapes. The obtained target shape skeleton has the similar topology of the source skeleton such as an equal number of joints, approximately identical joints' positions and hierarchy structure.

The rest of this paper is organized as follows. In Section II, we review previous works on shape correspondence and human skeleton extraction. The details of our proposed BSC are given in Section III. Firstly, we describe the model downsampling method and how to find the base points. Then this section focuses on how to select the base points and identify the similar points on the source and target shapes. In the end of this section, some results of correspondence are presented. Section IV describes the extraction of the source shape skeleton and generation of target shape skeleton through our shape correspondence approach and compare it with the direct skeleton extracting method. Our experimental results conducted on two benchmark datasets are presented in Section V, followed by conclusion and future work in Section VI.

\section{Related work}

The extraction of a model's skeleton should give enough information for the overall structure, while maintaining a certain level of details for the model. The pros and cons of various skeleton extraction methods and skeleton properties have been well documented in [19].

In the literature, various methods have been intensively studied for extracting skeletons 
from 3D shapes. Some methods concentrate on the pairwise skeleton correspondence [20]. Majority of the solutions focused only on the 1D curve-skeleton extraction from 3D objects which describes the abstract structure of the model [21] although some techniques try to compute a joint-based skeleton of the model [22]. The skeleton extracted by these methods generates satisfactory results for single input model. However, they suffer some limitations such as mismatching the location of skeletal joints with the real position of the model, failure to preserve the topology of the model, etc. For automatic extraction methods, the extracted joints hierarchy increases the complexity and fewer unwanted branches could be created. The generated skeletons through these methods also need to refine through skeleton pruning algorithms for every model.

The use of silhouettes as input to recognize human actions [23, 24] is based on the representation of the contour points from human silhouette. But the silhouette is obtained previously by extraction techniques, e.g. background subtraction and mostly from images. And many previous works use edges and silhouettes as pose descriptors [25, 26]. These methods use only image features and are not suitable for a strong dynamic model of human motion. The use of the same model for skeleton extraction and motion capture depends on a one-to-one correspondence between estimated and ground truth joints [27].

Base point means prominent feature point in this paper. We need them to be consistent on shapes with isometric deformations. Reuter and Peinecke [28] have proved that the Laplace-Beltrami eigenvalues as isometry-invariant shape descriptors could be used to recognize the isospectral models dubbed as "Shape-DNA". By the decomposition of the Laplace-Beltrami operator, Reuter got the isometry-invariant signature of a manifold from the eigenfunction corresponding to the first few smallest non-zero eigenvalues. But the "Shape-DNA" is a global feature. The heat diffusion on a manifold is the diffusion process whose infinitesimal generator is the Laplace-Beltrami operator[29]. Coifman and Lafon $[29,30]$ have introduced the diffusion processes on manifold which formed a base for the recent studies about the diffusion geometry in shape analysis. Sun and Ovsjanikov [31] proposed an intrinsic, multi-scale and robust shape descriptor, Heat Kernel Signature (HKS). This descriptor is based on the physical processes of heat propagation on a shape and also related to the diffusion geometry proposed by Lafon. It was obtained through the heat kernel of different time interval. HKS is efficiently computable and provides a multi-scale way to capture information about neighborhoods of a given point [31]. In this approach, when time period is small, the HKS takes more information from the close neighbors and catches the local features.

In shape correspondence, isometry is a very important clue for finding the final result. Fortunately, with the development of 3D data acquisition technology, the acquisition of parameterization-free shapes becomes popular and the data are almost in isometric. The BSC method in this paper is based on the invariance of geodesic distance with isometric deformations. To obtain the correspondence directly between two sets is an NP-hard problem. Many researchers have proposed various methods to simplify it and construct some models based on some descriptors [32, 33] and metric structure of shape [34-36]. H. Zhang et al. [37] proposed a deformation view of shape correspondence and proposed a deformation-driven method. Yusuf Sahillioğlu et al. [38] proposed a greedy optimization on the isometry cost model, and then took different algorithms in [39-41] to detect the correspondence. This approach has solved the correspondence problem with greedy optimization, but it needs a good initialization in order to avoid getting stuck with local maxima. Instead, our approach is able to avoid this by solving a minimum cost max flow problem.

\section{Base-points-driven Shape Correspondence}

In this section we first review the downsampling method, Farthest Points Sampling (FPS) [42]. How to represent these down sampled points and eliminate the effects of nonrigid deformations between them are major challenges for obtaining the plausible result of 


\subsection{Downsampling methods}

In order to improve the efficiency of the algorithm, a limited number of points have been required under a certain magnitude. A downsampling algorithm FPS has been used to decimate the points. FPS provides almost evenly spaced sampling, the next sample is placed in the center of the largest empty disk on surface. In other words, the next sample is placed at a point that is farthest from the previous samples.

In the FPS procedure, the points at the anchors (e.g. the feet and the hands of human or animal shapes) will be selected first. Various anchor points of each isometric shape will be corresponded and selected by FPS which obtains a well-separated covering net of the shape. By taking the advantage of FPS, we have obtained subsets of the shape for our shape correspondence method.

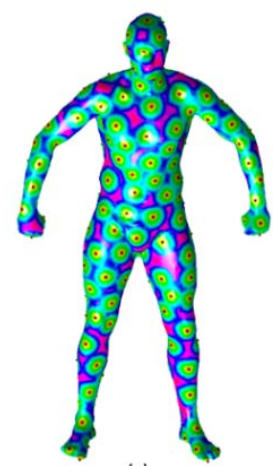

(a)

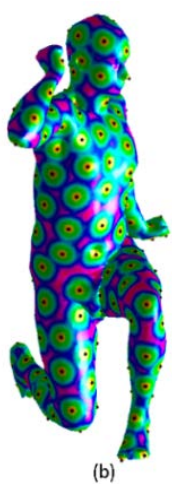

(b)

Figure 1 Results of the FPS downsampling on two different shapes with 200 points

\subsection{New shape representation}

For a 3D surface $\mathbf{S}(u, v)$, which is a 2D manifold surface, a geodesic metric $d_{\text {geo }}(x, y)$ is defined on it, where $\mathrm{x}, \mathrm{y}$ are two points on the surface. Three or more points which are not on a same geodesic path are selected as the base points: p1, p2, and p3. Then arbitrary point 0 on the surface will have an exact location. The $d_{\text {geo }}\left(p_{0}, p_{i}\right)$ has been computed, where $i=1,2,3$. These three geodesic distance values have been taken to be the new coordinates for all the points on the surface $P 0\left(d_{\text {geo }}(p 0, p 1), d_{\text {geo }}(p 0, p 2), d_{\text {geo }}(p 0, p 3)\right)$ in a 3D Euclidian space.

After calculating a point p, we compute the geodesic distances from this point, which forms a field on the surface, through the geodesic function $f(q)=d_{\text {geo }}(q, p)$. Every point only has one value. All the same value points are on a close curve, similar to the circular contour on the plane in Euclid space.

In the Euclidean geometry, it has well known three points, which are not on the same curve, can decide one and only one plane. This can be described by the intersection point of three circles, which takes the base points as the centers and the distances from the base points as the radius respectively as shown in Figure 2. For each point of the surface, we can find the unique group of circles as the coordinate system. 


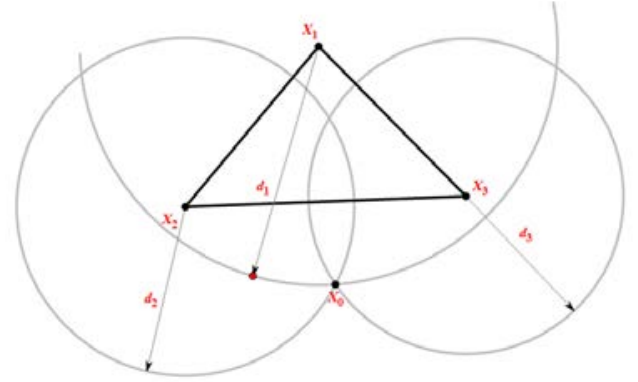

Figure 2 Any point can be represented by the intersect point of three circles centered by three base points.

We extend this method to the manifold surface with the geodesic metric. The geodesic distance is selected to replace the Euclid distance, and geodesic contour which is a closed curve is used to replace the circle, as illustrated in Figure 3.

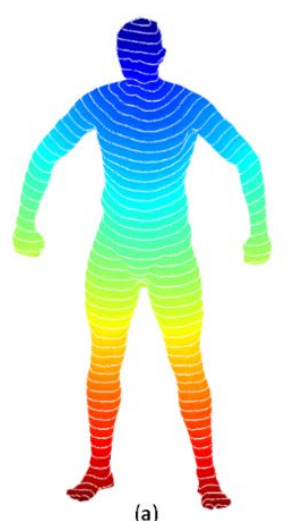

(a)

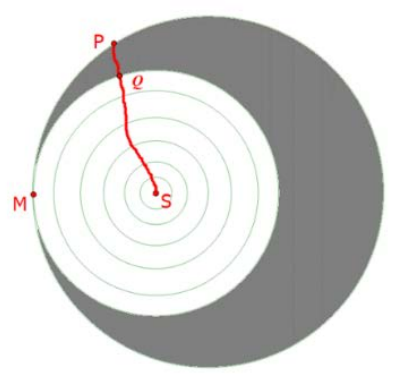

(b)

(a) Geodesic contours from a source point on a human pose shape. (b) Illustration on why these contours do not intersect when the two geodesic contour intersect at point $\mathrm{M}$.

Figure 3 Example of geodesic

In Figure 3(a), the geodesic contour is some close curves and there is no intersection point between any two geodesic contours because of a unique distance between any point and the source point. If there are two geodesic contours intersect at one point $\mathrm{M}$, all the points on the two contours have the same geodesic distance. In Figure 3(b), considering a point $\mathrm{P}$ on the outer contour, the geodesic path from the source $\mathrm{S}$ to point $\mathrm{P}$ pass through the area, is between the two contours. When it enters into this area, there will be an intersect point $\mathrm{Q}$ within the inner contour. However, the points $\mathrm{P}$ and $\mathrm{Q}$ have the same distance. So there is no intersecting point between any two geodesic contours.

Three base points are selected, all the points on the surface can be defined by geodesic distances between base points. Under this situation, if base points of two shapes are given in order respectively, a sequence of distance values can be used to represent a point on the surfaces. Because geodesic distances are unique, the sequence will also be unique for one point. It's very important to define these consistent base points on different pose shapes. The following section presents our approach to find the same base points on the source and target shapes using an intrinsic point feature Scale-invariant Heat Kernel Signature (SIHKS) [43].

\subsection{Heat Kernel and Heat Kernel Signature}

3D shapes representations of human posture shapes are convenient in applications such as rendering and visualization. But it is not suitable, at least in a direct way, for many other 
situations including human motion comparison, motion correspondence, shape estimation, and recognition. In these applications, the pose shapes are considered to be similar if there exist rigid or isometric transformations between them.

Considering the non-rigid deformations of these posture models, this makes it difficult to find their correspondence. Therefore we need a method to eliminate the influence of the deformations. So the HKS as the intrinsic features is chosen to find the base points.

Let $M$ be a compact Riemannian manifold without boundary, the amount of heat at a point $p \in M$ at time $\mathrm{t}$ is defined by $u(p, t): M \times \mathfrak{R}^{+} \rightarrow \mathfrak{R}^{+}$. So at time 0 , the heat at every point could be represented as the function $f: M \rightarrow \mathfrak{R}^{+}$. Moreover the diffusion of heat on $M$ is governed by the heat equation as follows:

$$
\left(\Delta_{M}+\frac{\partial}{\partial t}\right) u(p, t)=0
$$

Here $\Delta_{M}$ is a Laplace-Beltrami operator of $M$. If the heat distribution $f$ at time $t$ is given by the heat operator $H_{t}$, then the following equations are satisfied:

$$
\lim _{t \rightarrow 0} H_{t} f=f \text {, and } u(p, t)=H_{t} f(p)
$$

And these two operators have the relation $H_{t}=e^{-t \Delta_{M}}$.

The heat kernel is a function as described in the following equation:

$$
k_{t}(p, q): \mathfrak{R}^{+} \times M \times M \rightarrow \mathfrak{R}^{+}
$$

It satisfies $H_{t} f(p)=\int_{M} k_{t}(p, q) f(q) d q$ for all $p \in M$ and measures the amount of heat transferred from $p$ to point $q$ in time $t$. According to the eigen-decompositon of LaplaceBeltrami operator and the relation of $\Delta_{M}$ and $H_{t}$, the spectral expansion of heat kernel on any compact manifold $\mathrm{M}$ has the following form:

$$
k_{t}(p, q)=\sum_{i=0}^{\infty} e^{-t \lambda_{i}} \varphi_{i}(p) \varphi_{i}(q)
$$

Here $\lambda_{i}$ and $\varphi_{i}$ are $i$-th eigenvalue and its corresponding eigenfunction of LaplaceBeltrami operator respectively. From Eq. (3), we can see that, the heat kernel is symmetric $k_{t}(p, q)=k_{t}(q, p)$. The HKS is also isometry-invariant for the shapes with isometric transformations. Inversely if two shapes have the same HKS, then they are isometric.

$H K S(p): \mathfrak{R}^{+} \rightarrow \mathfrak{R}, H K S(p, t)=k_{t}(p, p)$ is exactly the Heat Kernel Signature defined on the point $\mathrm{p}$ of the manifold $\mathrm{M}$. It could be represented as:

$$
k_{t}(p, p)=\sum_{i=0}^{\infty} e^{-t \lambda_{i}} \varphi_{i}(p)^{2}
$$

As a local shape descriptor HKS has also many properties such as multi-scale property especially sampled at a finite set of time $t_{1}, \cdots, t_{n}$ :

$$
H K S(p)=\left(k_{t_{1}}(p, p), k_{t_{2}}(p, p), \cdots k_{t_{n}}(p, p)\right)
$$

In order to deal with global and local scaling transformations, we need a scale-invariant method which could be achieved by [43-45]. In this paper, the Scale-invariant Heat Kernel Signature has been employed for defining the base points. According to Bronstein [43], shape scaling factors could be removed by the logarithmically sampling, discrete derivative and discrete-time Fourier transforms. This approach created scale-invariant feature descriptors, and the SIHKS extend the heat kernel signature to deal with global and local scaling transformations. By means of the scale and pose invariant properties of SIHKS, we could find the consistent base points on two shapes with isometric deformations by the local extreme of the SIHKS. Figure 4 shows some results of the base points (marked with pink balls) on the same person with different poses. These points are taken from the local maximum SIHKS in the range of a fixed geodesic distance on shapes. 


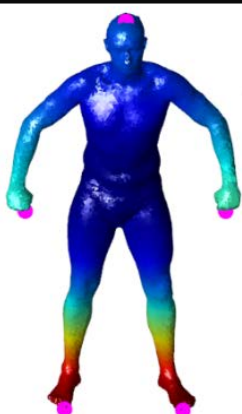

(a)

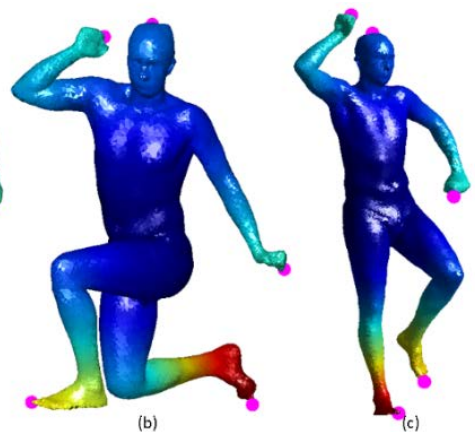

(a) Base points (pink balls) on a standing pose. (b) Base points on the squatting pose. (c) Base points on the dancing pose.

Figure 4 Example of base points on different shape poses

\subsection{Identification of similar points}

In this subsection, we describe the method to compute the correspondence of points on the source and target shape. The base points are selected, and they have been matched to each other. Now that the geodesics from these base points are computed, we take the order of the base points to be the order of new coordinate in Euclidian embedding space. The geodesic distances matrix $M_{1}$ and $M_{2}$ have been computed by using the fast marching algorithm [46].

After embedding the shape into Euclidean space through setting the matched and ordered points of original shapes as base points, two new shapes are achieved which have the same location and the same surface, as shown in Figure 5. The correspondence problem then becomes to find the closest pairs of points between two point sets.

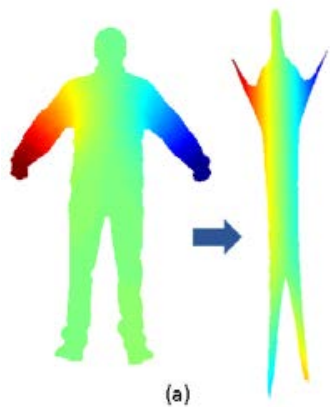

(a)

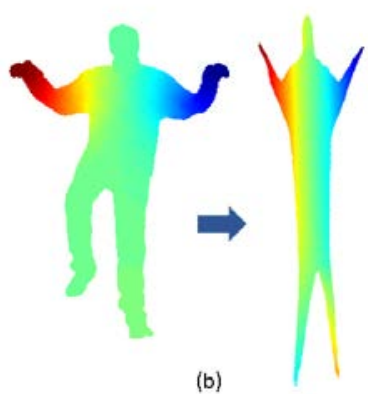

(b)

(a) Standing pose and its representation. (b) Crane pose and its representation.

Figure 5 Embed two new shapes into the same Euclidean space

Let's denote the $M_{1}\left(\mathrm{~V}_{1}, \mathrm{~F}_{1}\right)$ and $M_{2}\left(\mathrm{~V}_{2}, \mathrm{~F}_{2}\right)$ as the source shape and the target shape respectively, where $V_{i}(i=1,2)$ is the set of new points, which can be written as $\mathbf{v}\left(v_{1}, v_{2}, v_{3}, \cdots, v_{k}\right), k$ is the number of the base points. $F_{i}$ is the face from the original shape, i.e. the topology of the original and the new shape are same. Now we just find the nearest points between the two sets. The greedy strategy can be taken to find the nearest point from $M_{2}$ for each point in $M_{1}$. However, it just meets the local optima. Our aim is to find a map $\Psi\left(v_{1}\right): v_{1} \in M_{1} \rightarrow v_{2} \in M_{2}$, which is given below:

$$
\Psi(v)=\underset{v_{i} \in M_{1}, \Psi\left(v_{i}\right) \in M_{2}}{\operatorname{argmin}} \sum_{i=1}^{N} L_{2}\left(v_{i}, \Psi\left(v_{i}\right)\right)
$$


Here $L_{2}\left(v_{i}, \Psi\left(v_{i}\right)\right)$ is the $L_{2}$ distance and $N$ is the number of $M_{1}$. Now this problem changes to an optimization problem, which could be solved by the following minimum-cost maximum flow problem. The minimum-cost flow [47] is an important and typical problem in the field of graph theory, and is the core of network optimization problems.

This problem can be described as follows. Giving a flow network, that is, a directed graph $G=(V, E)$, with source point $s \in V$ and a target point $t \in V$, each edge $e(i, j) \in E$ has an associated cost $a_{i j}$ that denotes the cost per unit flow on that edge. A flow $f_{i j} \geq 0$ and a capacity $c_{i j}>0$ are associated with every edge. We can send a flow $f$ from $s$ to $t$.

The minimum-cost flow problem is an optimization model formulated as follows:

$$
\text { Minimize } \sum_{(i, j) \in E} a_{i j} f_{i j}
$$

Subject to $f_{i j} \leq c_{i j}, f_{i j}=-f_{j i}, \sum_{j \in V} f_{i j}=0$ for all $i \neq s, i \neq t$ and $\sum_{j \in V} f_{s j}=\sum_{i \in V} f_{i t}=d$.

Based on this, if the point set, except $s$ and $t$, can be separated into two sets with the edges, we call this graph is a bipartite graph. Then with a weight to every edge, we get the minimum weight graph, which will be used in our method to solve the correspondence problem.

\subsection{Correspondence results}

Some of our correspondence results are illustrated in Figure 6. We represent the correspondence between each pair of shapes by the lines. The distances are computed between each correspondence points in $k$ dimensional Euclidian space (in our experiment $k=5$ ). After that we take the average of these distances as the measure standard to test the quality of the result.

Figure 6 also shows the sparse and dense correspondence results. Sometimes the coarse correspondence may lead to less computation time, but this will result in reduced accuracy for skeleton extraction. So the dense correspondence is more suitable for our case.

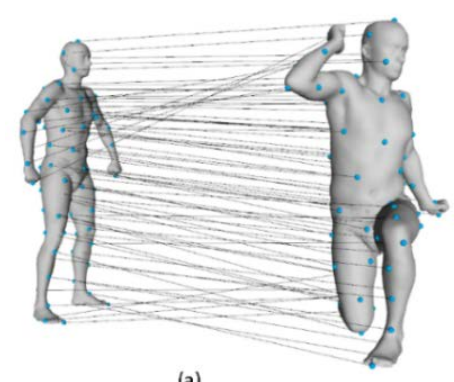

(a)

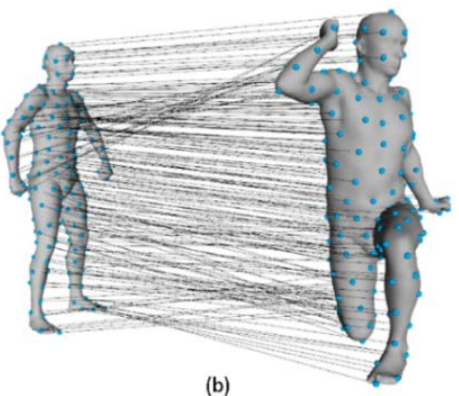

(b)

(a) Sparse result. (b) Dense result.

Figure 6 Result of the correspondence

\section{Skeleton extraction through shape correspondence}

After the establishment of the correspondence between target and source shapes, we want to extract the target shape skeleton by using the source one. The process of extracting target shape skeleton consists of two steps. At first, a skeleton has been extracted from one of the correspondence shape (source shape). Then a similar plausible skeleton is generated of target shape based on the source shape skeleton. The obtained target skeleton maintains equal 
number of joints and preserves similar hierarchy of the source skeleton joints.

\subsection{Source shape skeleton extraction}

The suitable source shape skeleton has been extracted using the method in [10]. At first the source shape is contracted through Laplacian-based mesh contraction which converts the geometric model into the skeletal shape of the input model. The contracted mesh consists of approximate zero-volume that is visually skeletal representation of that shape. This contraction process preserves the original topology and maintains the real shape of the model. The linear system of vertex positions is iteratively solved during the mesh contraction by using Eq. (8) below:

$$
\left[\begin{array}{l}
W_{C} L \\
W_{A}
\end{array}\right] V^{\prime}=\left[\begin{array}{l}
0 \\
W_{A}
\end{array}\right]
$$

where $W_{c}$ and $W_{A}$ are the constraints of contraction and attraction respectively. The contraction and attraction constraints have been minimized through quadratic energy function by using Eq. (9).

$$
\left\|W_{C} L V^{\prime}\right\|^{2}+\sum_{i} W_{A, i}^{2}\left\|v_{i}^{\prime}-v_{i}\right\|^{2}
$$

In this equation the first part relates to contraction constraints and the second part describes the attraction constraints. $W_{A, i}$ is the attraction weight for every point $i$ after each iteration. $L$ is the $n \times n$ Laplace operator with elements, and $V$ 'represents the contracted vertex of the source shape. In order to get more accurate results of the skeleton extraction, we may apply minimum user intervention.

The contracted mesh is then converted into hierarchical joint-based skeleton through topological thinning and geometry refinements. In topological thinning we have applied edgecontraction operation on the contracted mesh to collapse the unnecessary edges until to create the $1 \mathrm{D}$ curve-skeleton of the shape. The edge-collapse process is applied repeatedly until all triangles in the contracted mesh are removed and the final curve-skeleton is obtained. The obtained 1D curve-skeleton is then refined into hierarchical joint-based skeleton through geometric refinements. The refinements include: computation of skeleton joints, identification of the root node, establishment of the hierarchy between skeleton joints and creation of the graph of skeleton joints. The extracted joint-based skeleton by implementing topological and geometric refinements on the contracted shape of the source model is given in Figure 7(b).

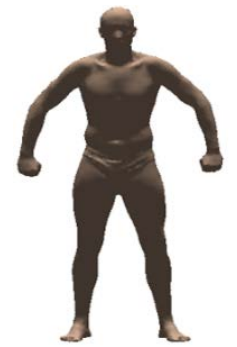

(a)

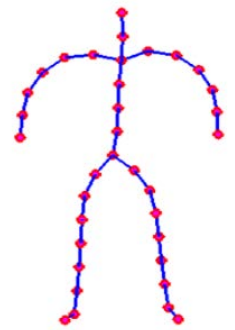

(b)

(a) Source shape. (b) Skeleton of the source shape.

Figure 7 Extraction of the source shape skeleton

\subsection{Consistent skeleton generation for target shape}

The computed skeleton of the source shape is then used to extract the consistent 1D curve-skeleton of the target shape through computed surface correspondence of the source to the target shape. The joints of the source skeleton are used to calculate the skeleton-vertex 
correspondence of source shape through the matrix $D . D$ is the shortest distance between every joint of the source skeleton with its shape point described in Eq. (10). Denote the source model as $P$ and size of the model points as $m$. The extracted skeleton $S$ consists of $n$ joints. The skeleton to shape surface relations is calculated as:

$$
\begin{gathered}
D_{i j}=\left\|P_{i} S_{j}\right\|, i=1,2, \cdots, m, j=1,2, \cdots, n \\
\left\|P_{i} S_{j}\right\|=\sqrt{\left(P_{i 1}-S_{j 1}\right)^{2}+\left(P_{i 2}-S_{j 2}\right)^{2}+\left(P_{i 3}-S_{j 3}\right)^{2}}
\end{gathered}
$$

The closest shape vertex $E$ of source skeleton joints is determined by distance matrix $D$, $E_{i}=D_{I_{i}}$, where $I_{i}$ is minimum distance of the every skeleton joint from its associated shape vertex.

The projected nodes of the target shape skeleton have been estimated using BSC shape correspondence and skeleton-surface relationship $E$ of the source shape. Our BSC approach computes one to one correspondence between the source and target shapes points. Thereafter, the similar points of the target shape are grouped into one group (cluster) based on $E$. Let the target shape points are $V$ composed of $k$ group corresponding such that $v_{1} U v_{2} \cup \ldots \cup v_{k}=V$. Denoting the size of $j$-th group in target mesh points as $n_{j}(j=1,2 \ldots, k)$, we calculate the center of each group $\bar{v}_{j},(j=1,2 \ldots, k)$ of the target mesh vertex using Eq. (12) as:

$$
\bar{v}_{j}=\frac{\sum_{i=1}^{n_{j}} v_{i j}}{n_{j}}, j=1,2, \ldots, k
$$

The prototype (center) of each group is computed of target shape that represents the extracted nodes of the target shape skeleton $T=\left\{\bar{v}_{i}, \bar{v}_{j} \cdots, \bar{v}_{k}\right\}$ as shown in Figure 8. To connect the target shape nodes, a cyclic connection between nodes has been constructed based on shape correspondence and using rings of share vertices in target shape. To compute the connection between target shape nodes, at first, we compute the connected rings of the shape vertices.

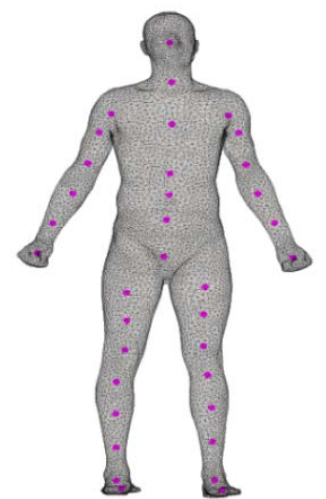

Figure 8 The extracted nodes of the target shape

Nearest neighbor search (NNS) algorithm is applied to find the closest points in the shape for computing one-rings of mesh point neighbors. Numerous solutions in computational geometry have been proposed for NNS problems such as linear search which calculates the distance from a query to every data point, local sensitive hashing (make buckets of data based on a distance metric), greedy search (construct graph of the points from query to its neighborhood). We use the space-partitioning algorithm for NNS based on K-d tree data structure [48]. K-d tree algorithm is based on iteratively division of the search space into two regions. The K-nearest neighbor of mesh points is computed by using $\mathrm{k}$ - $\mathrm{d}$ nearest neighbors 
search. At first, we compute an approximate neighborhood of target shape $V_{i}$ by calculating its $k$ nearest neighbors $N_{k}\left(V_{i}\right)$. The approximate neighbors are projected on a tangent plane defined by their principal components analysis. A planar Delaunay triangulation is constructed and defines one-ring neighbors of mesh points which are used to build the connection between target mesh nodes. The parameter required to define the number of nearest neighbors is $k=0.012$. We use this parameter to estimate the tangential plan and bounding minimum and maximum values (neighbors) in the range of [8:30]. A similar value of $k$ has been used throughout experiments in all shapes for computing the rings of approximate neighbors of the mesh points. In cyclic connection, the value of connected nodes with its neighbor nodes is equal to 1 . The generated joint-skeleton of the target shape that is approximately similar to source shape skeleton present in Figure 9 (b). In addition, the obtained skeleton from the target shape also generates skeleton joints to surface points mapping that can be directly applicable to mesh skinning deformation.

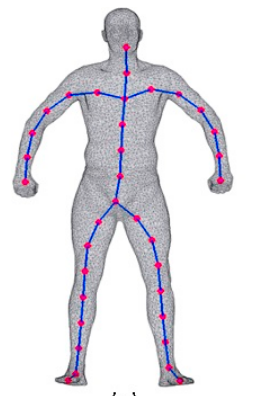

(a)

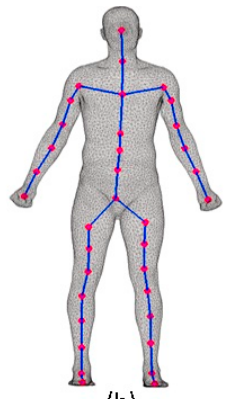

(b)

(a) Source shape, (b) Consistent skeleton of target shape.

Figure 9 Consistent skeleton extraction based on source shape skeleton

\section{Results and Discussions}

Although, the extraction of a $1 \mathrm{D}$ curve-skeleton through mesh contraction gives a satisfactory skeleton for single shape, but it does not always generate a robust skeleton for every pose of the same shape. In Figure 10 we compare the extracted skeleton of the target shape skeleton from two approaches: one is the mesh contraction by applying topological and geometric refinements and the other is BSC. In Figure 10(a) it can be seen that the target shape skeleton in some extent is off center and has the sharp bend of the bones. A post processing is needed to correct the position of the skeletal joints. On the contrary, our result shown in Figure 10(b) is much better. The resulting skeleton remains consistent over the poses change of the source shape and generates analogue skeletons of the target shape as shown in Figure 9. 


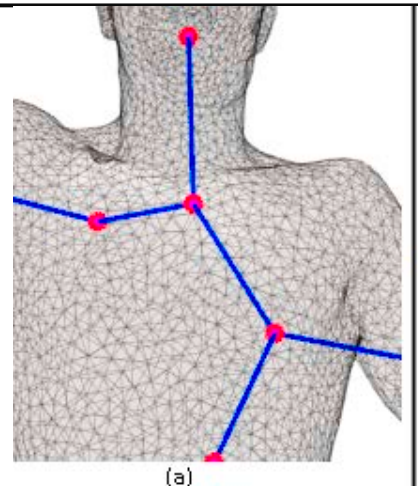

(a)The result of mesh contraction.

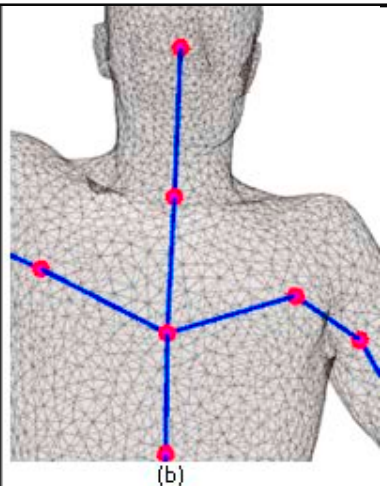

(b)

Figure 10 Comparison of our method and mesh contraction

Figure 11 gives more results of the skeleton extraction and comparisons of our approach to the direct skeleton extraction of the target shape. We test our approach on two different datasets: Vlasic et al [49] and SCAPE: Shape Completion and Animation of People [50]. In both datasets each single person performs multiple motions. The shapes in first column of Figure 11 are the source shapes with their extracted skeleton. The source shape skeletons are extracted through geometric contraction, topological and geometric refinements. Automatic generated skeletons of the target shapes by using BSC are present in second and fourth columns of Figure 11. The obtained skeleton of the target shape is consistent with the source shape skeleton. The skeletons of the target shapes have an equal number of joints as the source shape. They are well-centered and have similar hierarchy of the source shape skeleton joints.

\begin{tabular}{|c|c|c|c|c|}
\hline $\begin{array}{c}\text { Skeleton of } \\
\text { source }\end{array}$ & $\begin{array}{c}\text { Skeleton of } \\
\text { target from BSC }\end{array}$ & $\begin{array}{c}\text { Skeleton of } \\
\text { target from } \\
\text { direct extraction }\end{array}$ & $\begin{array}{c}\text { Skeleton of } \\
\text { target from BSC }\end{array}$ & $\begin{array}{c}\text { Skeleton of } \\
\text { target from } \\
\text { direct extraction }\end{array}$ \\
\hline & &
\end{tabular}




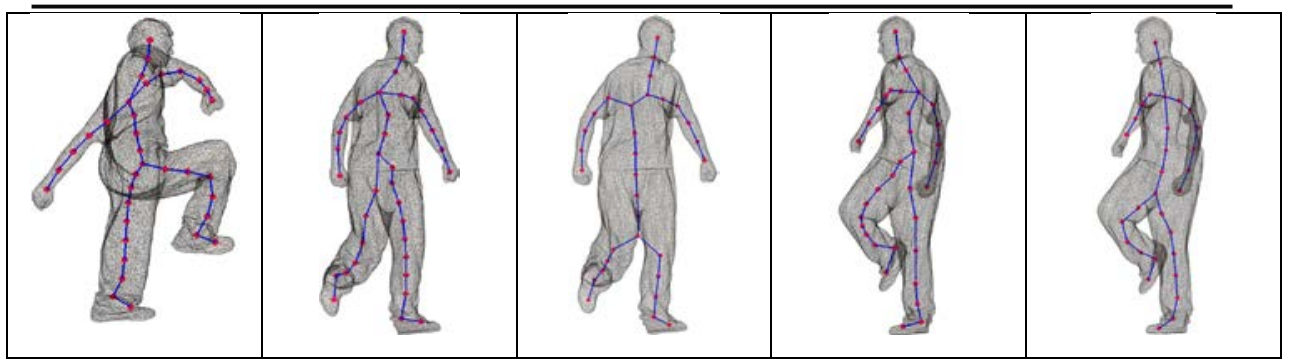

Figure 11 Comparisons with other results

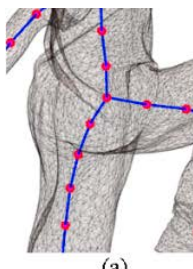

(a)

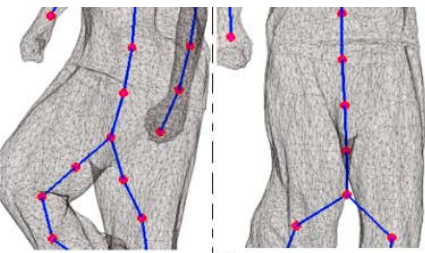

(b)
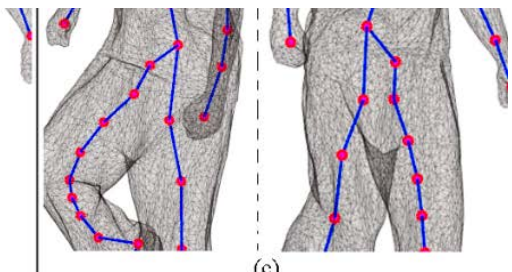

(c)

(a) Source skeleton (b) Result of direct skeleton extraction method (c) Result of BSC

Figure 12 Consistency of skeleton point position

Majority of the skeletonization methods may generate different skeletons for different postures of the same shape. As shown in Figure 11, the direct skeletons extraction approach obtains different skeletons of the target shape with different poses. Those skeletons have unequal number of joints, different joints hierarchy and do not match satisfactory position of joints with its original shape. Based on BSC, our approach is able to extract consistent skeletons of target shapes which are different poses of the source shape. And our approach does not require any topological, geometrical and embedding refinement for every poses of same shape. There is no need to set approximation parameters for extracting a skeleton of same shape with different poses. The results in Figure 12 demonstrate the robustness of our approach. The source skeleton joints Figure 12(a) and our result Figure 12(c) are consistent despite of the position of the model's pants. However, the result Figure 12 (b) obtained through the direct skeleton extraction is not accurate due to the pants' position in the target pose is lower than that in the source shape.

\section{Conclusion and future work}

In this paper, we have proposed an automatic and pose-invariant approach to extract the skeleton of an articulated human model based on BSC. We apply BSC to find the consistency of geometric information between shapes. Based on the corresponding relationship between the source and target shapes, the plausible skeleton of the target shape is extracted. Our approach supports the reusability of the source skeleton from the same model. It does not require any pre-processing to shapes, such as downsampling, mesh contraction, simplification and etc. In the proposed solution, the skeletons of different poses of the target shapes are computed instead of single shape.

The robustness of our proposed method is demonstrated through our tests on two wellknown datasets. Experiments have shown that our algorithm produces both effective and accurate skeletons of the target shapes. Compared with the directly extracting method, our approach performs better in term of consistency such as equal number of skeleton joints and similar hierarchy between skeleton joints for a variety of target shapes. Furthermore, the skeleton generated by our approach is efficient because it does not require post-processing such as topological and geometric refinements. In the future we will investigate and study 
more efficient and robust correspondence method so that the accuracy and consistency of the skeleton extraction can be improved further.

\section{Acknowledgments}

The research is partially supported by National Natural Science Foundation of China (No.61170170 and 61170203) and the National Key Technology Research and Development Program of China (2012BAH33F04).

\section{References}

1. Golovinskiy A, Funkhouser T (2009) Consistent segmentation of 3D models. Computers \& Graphics 33 (3):262-269

2. Smeets D, Hermans J, Vandermeulen D, Suetens P (2012) Isometric deformation invariant 3D shape recognition. Pattern Recognition 45 (7):2817-2831. doi:http://dx.doi.org/10.1016/j.patcog.2012.01.020

3. Mahmoudi M, Sapiro G (2009) Three-dimensional point cloud recognition via distributions of geometric distances. Graphical Models 71 (1):22-31

4. Lian Z, Godil A, Bustos B, Daoudi M, Hermans J, Kawamura S, Kurita Y, Lavoué G, Van Nguyen H, Ohbuchi R (2011) SHREC'11 Track: Shape Retrieval on Non-rigid 3D Watertight Meshes. 3DOR 11:79-88

5. Tam GK, Cheng Z-Q, Lai Y-K, Langbein FC, Liu Y, Marshall D, Martin RR, Sun X-F, Rosin PL (2013) Registration of 3D point clouds and meshes: A survey from rigid to nonrigid. Visualization and Computer Graphics, IEEE Transactions on 19 (7):1199-1217

6. Dey TK, Li K, Luo C, Ranjan P, Safa I, Wang Y Persistent Heat Signature for Pose - oblivious Matching of Incomplete Models. In: Computer Graphics Forum, 2010. vol 5. Wiley Online Library, pp 1545-1554

7. Bandouch J, Engstler F, Beetz M (2008) Accurate human motion capture using an ergonomics-based anthropometric human model. In: Articulated motion and deformable objects. Springer, pp 248-258

8. Hahmann S, Belyaev A, Busé L, Elber G, Mourrain B, Rössl C (2008) Shape interrogation. In: Shape Analysis and Structuring. Springer, pp 1-51

9. Li X, Woon TW, Tan TS, Huang Z Decomposing polygon meshes for interactive applications. In: Proceedings of the 2001 symposium on Interactive 3D graphics, 2001. ACM, pp 35-42

10. Tagliasacchi A, Zhang H, Cohen-Or D Curve skeleton extraction from incomplete point cloud. In: ACM Transactions on Graphics (TOG), 2009. vol 3. ACM, p 71

11. Le BH, Deng Z (2014) Robust and accurate skeletal rigging from mesh sequences. ACM Transactions on Graphics (TOG) 33 (4):84

12. Baran I, Popović J Automatic rigging and animation of $3 \mathrm{~d}$ characters. In: ACM Transactions on Graphics (TOG), 2007. vol 3. ACM, p 72

13. Cornea ND, Demirci MF, Silver D, Shokoufandeh A, Dickinson SJ, Kantor PB 3D object retrieval using many-to-many matching of curve skeletons. In: Shape Modeling and Applications, 2005 International Conference, 2005. IEEE, pp 366-371

14. Kin - Chung Au O, Tai CL, Cohen - Or D, Zheng Y, Fu H Electors voting for fast automatic shape correspondence. In: Computer Graphics Forum, 2010. vol 2. Wiley Online Library, pp 645-654

15. Yan H-B, Hu S-M, Martin RR, Yang Y-L (2008) Shape deformation using a skeleton to drive simplex transformations. Visualization and Computer Graphics, IEEE Transactions on 14 (3):693-706

16. Au OK-C, Tai C-L, Chu H-K, Cohen-Or D, Lee T-Y Skeleton extraction by mesh contraction. In: ACM Transactions on Graphics (TOG), 2008. vol 3. ACM, p 44

17. Kravtsov D, Fryazinov O, Adzhiev V, Pasko A, Comninos P Controlled Metamorphosis Between Skeleton - Driven Animated Polyhedral Meshes of Arbitrary Topologies. In: Computer Graphics Forum, 2014. vol 1. Wiley Online Library, pp 64-72

18. Wong S-K, Yang J-A, Ho T-C, Chen Y-C, Chuang J-H (2012) A skeleton-based approach for shape correspondence. Paper presented at the Proceedings of the 11th ACM SIGGRAPH International Conference on Virtual-Reality Continuum and its Applications in Industry, Singapore, Singapore,

19. Cornea ND, Silver D, Min P (2007) Curve-skeleton properties, applications, and algorithms. Visualization and Computer Graphics, IEEE Transactions on 13 (3):530-548

20. Zheng Q, Sharf A, Tagliasacchi A, Chen B, Zhang H, Sheffer A, Cohen-Or D (2010) Consensus Skeleton for Non-rigid Space-time Registration. Computer Graphics Forum 29 (2):635-644. doi:10.1111/j.14678659.2009.01633.x

21. Jiang W, Xu K, Cheng Z-Q, Martin RR, Dang G (2013) Curve skeleton extraction by coupled graph contraction and surface clustering. Graphical Models 75 (3):137-148

22. Ma J, Choi S (2014) Kinematic skeleton extraction from 3D articulated models. Computer-Aided Design 46:221-226

23. Chaaraoui AA, Climent-Pérez P, Flórez-Revuelta F (2013) Silhouette-based human action recognition using sequences of key poses. Pattern Recognition Letters 34 (15):1799-1807. doi:http://dx.doi.org/10.1016/j.patrec.2013.01.021 
24. Bandouch J, Jenkins OC, Beetz M (2012) A self-training approach for visual tracking and recognition of complex human activity patterns. International journal of computer vision 99 (2):166-189

25. Deutscher J, Reid I (2005) Articulated body motion capture by stochastic search. International Journal of Computer Vision 61 (2):185-205

26. Zhang W, Shang L, Chan AB (2014) A Robust Likelihood Function for 3D Human Pose Tracking.

27. Schwarz LA, Mkhitaryan A, Mateus D, Navab N (2012) Human skeleton tracking from depth data using geodesic distances and optical flow. Image and Vision Computing 30 (3):217-226. doi:http://dx.doi.org/10.1016/j.imavis.2011.12.001

28. Reuter M, Wolter F-E, Peinecke N (2006) Laplace-Beltrami spectra as 'Shape-DNA'of surfaces and solids. Computer-Aided Design 38 (4):342-366

29. Coifman RR, Lafon S (2006) Diffusion maps. Applied and Computational Harmonic Analysis 21 (1):5-30. doi:10.1016/j.acha.2006.04.006

30. Lafon SS (2004) Diffusion maps and geometric harmonics. Yale University,

31. Sun J, Ovsjanikov M, Guibas L A Concise and Provably Informative Multi - Scale Signature Based on Heat Diffusion. In: Computer Graphics Forum, 2009. vol 5. Wiley Online Library, pp 1383-1392

32. Yang Y, Günther D, Wuhrer S, Brunton A, Ivrissimtzis I, Seidel H-P, Weinkauf T Correspondences of persistent feature points on near-isometric surfaces. In: Computer Vision-ECCV 2012. Workshops and Demonstrations, 2012. Springer, pp 102-112

33. Berretti S, Werghi N, Del Bimbo A, Pala P (2013) Matching 3D face scans using interest points and local histogram descriptors. Computers \& Graphics 37 (5):509-525

34. Aflalo Y, Dubrovina A, Kimmel R (2013) Spectral generalized multi-dimensional scaling. arXiv preprint arXiv:13112187

35. Zhang M, Li F, Wang X, Wu Z, Xin S-Q, Lui L-M, Shi L, Wang D, He Y (2014) Automatic registration of vestibular systems with exact landmark correspondence. Graphical Models (0). doi:http://dx.doi.org/10.1016/j.gmod.2014.04.010

36. Jia T, Wang K, Wu Z, Zhao J, Xu P, Liu C, Zhou M Isometric Shape Matching Based on the Geodesic Structure and Minimum Cost Flow. In: Cyberworlds (CW), 2014 International Conference on, 6-8 Oct. 2014 2014. pp 122-129. doi:10.1109/CW.2014.25

37. Zhang H, Sheffer A, Cohen-or D, Zhou Q, Kaick OV, Tagliasacchi A (2008) Deformation-Driven Shape Correspondence. Computer Graphics Forum 27 (5):1431-1439. doi:10.1111/j.1467-8659.2008.01283.x

38. Sahillioğlu Y, Yemez Y 3d shape correspondence by isometry-driven greedy optimization. In: Computer Vision and Pattern Recognition (CVPR), 2010 IEEE Conference on, 2010. IEEE, pp 453-458

39. Sahillioğlu Y, Yemez Y (2011) Coarse-to-Fine Combinatorial Matching for Dense Isometric Shape Correspondence. Computer Graphics Forum 30 (5):1461-1470. doi:10.1111/j.1467-8659.2011.02020.x

40. Sahillioglu Y, Yemez Y (2012) Minimum-Distortion Isometric Shape Correspondence Using EM Algorithm. Pattern Analysis and Machine Intelligence, IEEE Transactions on 34 (11):2203-2215. doi:10.1109/TPAMI.2012.26

41. Sahillioğlu Y, Yemez Y (2014) Partial 3-D Correspondence from Shape Extremities. Computer Graphics Forum 33 (6):63-76. doi:10.1111/cgf.12278

42. Eldar Y, Lindenbaum M, Porat M, Zeevi YY (1997) The farthest point strategy for progressive image sampling. Image Processing, IEEE Transactions on 6 (9):1305-1315

43. Bronstein MM, Kokkinos I Scale-invariant heat kernel signatures for non-rigid shape recognition. In: Computer Vision and Pattern Recognition (CVPR), 2010 IEEE Conference on, 2010. IEEE, pp 1704-1711

44. Ovsjanikov M, Mérigot Q, Mémoli F, Guibas L (2010) One Point Isometric Matching with the Heat Kernel. Computer Graphics Forum 29 (5):1555-1564. doi:10.1111/j.1467-8659.2010.01764.x

45. Wang K, Wu Z, Xu P, Zhao J, Jia T, Shui W, Ali S, Zhou M Scale-Invariant Heat Kernel Mapping. In: Cyberworlds (CW), 2014 International Conference on, 6-8 Oct. $20142014 . \quad$ pp 114-121. doi:10.1109/CW.2014.24

46. Kimmel R, Sethian JA (1998) Computing geodesic paths on manifolds. Proceedings of the National Academy of Sciences 95 (15):8431-8435

47. Ahuja RK, Magnanti TL, Orlin JB (1993) Network flows: theory, algorithms, and applications.

48. Yianilos PN (1993) Data structures and algorithms for nearest neighbor search in general metric spaces. Paper presented at the Proceedings of the fourth annual ACM-SIAM Symposium on Discrete algorithms, Austin, Texas, USA,

49. Vlasic D, Baran I, Matusik W, Popović J (2008) Articulated mesh animation from multi-view silhouettes. ACM Transactions on Graphics (TOG) 27 (3):97

50. Anguelov D, Srinivasan P, Koller D, Thrun S, Rodgers J, Davis J Scape: shape completion and animation of people. In: ACM Transactions on Graphics (TOG), 2005. vol 3. ACM, pp 408-416 